\title{
El enlace faltante entre cadenas globales de producción y ciudades globales: el servicio financiero en Ciudad de México y Santiago de Chile****
}

\begin{abstract}
Since the 1980s, the economies of Mexico and Chile have passed through a profound process of globalisation. The result has been a nodalisation of the cities' economies, documented by their high concentration of command and control functions essential for economic globalization. In order to specify the relationship between global city formation and the deepening of global integration we draw on the findings of the global city and global commodity chain literature. Since advanced producer services have been identified as key actors in interlocking de-centralized production sites and urban networks we have analyzed the involvement of financial institutions in bond and share issues of the 50 top ranked Mexican and Chilean enterprises. Based on this initial empirical investigation we take a first step to specify how the two firm-based, trans-state networks - global city-and global production networks - relate.
\end{abstract}

Keywords: global cities, global commodity chains, globalization, Chile, Mexico

\section{Resumen}

Desde los años ochenta, las economías de México y Chile pasaron por un profundo proceso de globalización. En cuanto a las ciudades capitales de ambós paises, esta globalización implicó una nodalización, que se ve en una concentración alta de los funciones de gestión económica. Intentamos especificar la relación entre la formación de ciudades globales y la profundización de la integración global por medio de un estudio de casos prácticos de proveedores de servicios financieros. Usando los datos de transacciones del mercado de valores, analizamos la implicación de instituciones financieras (locales y globales) en emisiones de bonos y acciones de las 50 empresas mejor posicionadas de México y Chile. Con nuestra investigación empirica inicial de intermediarios de servicios financieros, que proveen servicios a las corporaciones más importantes en México y en Chile, damos el primer paso para especificar cómo se relacionan las dos redes trans-estatales basadas en empresas -las redes de ciudades globales y de producción.

Palabras claves: ciudades globales, cadenas globales de producción, globalización, Chile, México. 


\section{Introducción}

$\mathrm{D}$ esde los años '80, las economías de México y Chile pasaron por un profundo proceso de globalización que alteró sustancialmente el papel de ambas ciudades capitales. Mientras, durante la sustitución de las importaciones, la función de las ciudades había sido la integración del mercado nacional, mostraremos en este artículo que últimamente Ciudad de México y Santiago de Chile se dedican cada vez más a producir la integración global de aquellos sectores de la economía nacional que son competitivos en el mercado mundial. Esto implica la anudación de las economías de las ciudades, lo cual es documentado por una alta concentración de funciones de mando y control, esenciales para la globalización económica.

Recurriendo a la literatura referente a ciudades globales y cadenas globales de producción, sostenemos que las funciones centrales de mando y control, normalmente identificadas con empresas de servicios avanzados de producción, son actores claves en entretejer lugares de-centralizados de producción con la red urbana. Intentamos especificar la relación entre la formación de ciudades globales y la profundización de la integración global por medio de un estudio de casos prácticos de proveedores de servicios financieros. Usando los datos de transacciones del mercado de valores, analizamos la implicación de instituciones financieras (locales y globales) en emisiones de bonos y acciones de las 50 empresas mejor posicionadas de México y Chile. Con nuestra investigación empírica inicial de intermediarios de servicios financieros, que proveen servicios a las corporaciones más importantes en México y en Chile, damos el primer paso

Instituto de Geografía, Universidad de Hamburgo. E-mail: parnreiter@geowiss.uni-hamburg.de.

Instituto Proyecto Desarrollo Internacional, Universidad de Viena. E-mail: karin.fischer@univie.ac.at.

Instituto Proyecto Desarrollo Internacional, Universidad de Viena. E-mail: karen.imhof@univie.ac.at.

Este artículo fue elaborado en el marco del proyecto Transformación y procesos urbanos en Latino América financiado por el Fondo Austriaco de Ciencias (FWF), No. 14883. Agradecemos a Peter Taylor por poner a nuestra disposición la base de datos GaWC. Enviado el 6 de febrero de 2007, aprobado el 3 de agosto de 2007. para especificar cómo se relacionan las dos redes trans-estatales basadas en empresas -las redes de ciudades globales y de producción.

\section{La formación de ciudades globales y la profunda integración de México y Chile en la economía mundial}

El argumento principal del enfoque de ciudades globales es que los procesos de globalización originan una nueva forma de centralidad en la cual una cierta cantidad de ciudades emergen como lugares claves. Las ciudades globales articulan economías regionales y nacionales en la economía mundial, y así sirven de nudo de comunicaciones donde flujos de dinero, información, mercancías y migrantes se entrecruzan. De ahí que las ciudades globales sean tanto lugares de producción como lugares de comercio para mercancías específicas, o sea, servicios financieros y otros servicios avanzados de producción, esenciales para la integración global. Los flujos de estas mercancías vinculan las ciudades globales entre ellas; el resultado es la emergencia de la red de ciudades globales (Sassen, 1991; Taylor, 2004).

En artículos anteriores afirmamos que tanto Ciudad de México como Santiago estaban tomando funciones de ciudades globales desde los años 1980 (Parnreiter, 2002; 2003). Para confirmar la hipótesis de la formación de ciudades globales en México y en Chile, tres requerimientos tendrán que verse cumplidos. Debe estar probado, primero, que las dos economías progresivamente se han integrado en las cadenas globales de producción y comercio. Segundo, la globalización de las economías de México y Chile debe ser, a lo menos parcialmente, organizada, manejada y controlada desde su capital. Esto requiere un número considerable de proveedores de servicios avanzados de producción en las ciudades en cuestión. Tercero, las ciudades deben estar vinculadas con otras ciudades globales por medio de los flujos de servicios de producción, capital, información, etc. A continuación, ejemplificaremos cada uno de estos requerimientos.

La profunda integración de México y Chile en la economía mundial se muestra en un rápido incremento de las exportaciones, lo cual es fomen- 
tado por medio de acuerdos de libre comercio de ambos países, particularmente con los Estados Unidos y la Unión Europea (y adicionalmente la APEC, en el caso de Chile). Pero no solamente crece el valor anual de exportaciones, además muestran un cambio significativo en su estructura. En México, las exportaciones manufactureras llegaron en el año 2000 al 90\% de todas las exportaciones. Estas exportaciones, por lo general, están organizadas vía cadenas globales de producción, entendiendo que, aproximadamente, el $85 \%$ de las exportaciones mexicanas consisten en importaciones libres de impuestos que se procesan en México y después se re-exportan (Dussel Peters, 2000). Chile basó su orientación de exportaciones en las reservas abundantes de recursos naturales. Mientras el cobre sigue siendo el artículo de exportación más importante, las exportaciones notradicionales (mercancías agrícolas y mercancías industriales procesadas, como harina de pescado, fruta enlatada, vino embotellado y productos de madera) continuamente ganaron importancia desde mediados de los años ' 80 . El sector de exportación agro-alimenticio se ha integrado progresivamente en las cadenas globales de mercancías que son controladas y manejadas por corporaciones transnacionales (CTN) (Gwynne, 1999).

Este hecho nos lleva a una segunda característica del cambio: una economía orientada hacia el mercado mundial. Debido a la privatización y a una reestructuración industrial extendida, las CTN ganaron una creciente influencia. En México, las CTN encabezan las cadenas globales de producción en la industria automotriz, electrónica, computacional y textil, y, además, del sector financiero. En Chile, las CTN dominan el sector agro-alimenticio. Las telecomunicaciones, energía y servicios financieros son generalmente trans-nacionalizados, con proveedores globales de servicios en búsqueda de acceso al mercado regional. Conglomerados en propiedad de capital privado chileno, siguen afirmando su posición, aunque los joint ventures con inversionistas extranjeros llegaron a ser la práctica normal y corriente (Fazio, 2000).

La presencia fuerte de CTN se refleja en el volumen y la dirección de inversiones extranjeras directas (IED). En México, la inversión extranjera directa anual creció ocho veces entre 1980 y 2000, con los sectores de transporte, de telecomunicaciones y de servicios financieros como perceptores principales. En Chile, los flujos de entrada de IED fueron 30 veces más grandes en el año 2000 comparado con 1995. El sector que más recibió es el minero, seguido por transporte, comunicaciones, servicios y electricidad.

La orientación creciente de las economías mexicanas y chilenas hacia el mercado mundial es, al menos parcialmente, organizada desde las dos capitales. Ciudad de México experimentó una profunda transformación de su base productiva bajo un nuevo régimen de desarrollo. Desarrollando un alto coeficiente de especialización local en servicios, la ciudad dejó de ser especializada en manufactura. Cifras de empleo subrayan este argumento: casi la mitad del empleo nacional en servicios (bienes raíces, financieros y profesionales) está concentrado en el área metropolitana, representando el sub-sector del mercado de empleo formal urbano que más está creciendo.

La concentración de sedes principales de compañías fortalece, además, el argumento para la formación de una ciudad global. Un análisis de la distribución regional de sedes principales de las compañías más importantes que operan en México, muestra que Ciudad de México no solo es el lugar donde más compañías tienen su centro de comando. Mejor dicho, el descubrimiento interesante es que mientras más grande es el volumen de venta de una empresa y más fuertes sus vínculos con la economía global en términos de exportaciones, importaciones y capitalización extranjera, más probable es que su sede principal esté ubicada en la parte interior de Ciudad de México, en el Destrito Federal. La distribución regional de IED apunta en la misma dirección. Es notable que el Distrito Federal haya mantenido un nivel muy alto $(60 \%)$ de todas las IED durante los últimos trece años, a pesar del término de privatizaciones importantes y pese al crecimiento significativo de IED dirigidas hacia las zonas de procesamiento de exportaciones, la denominada industria maquiladora (Parnreiter, 2002)

Los cambios en la estructura económica de Santiago de Chile comenzaron a principios de los 
años '70. Entre los años 1960 y 1990, el empleo en la manufactura sufrió pérdidas graves, mientras que los servicios aumentaron sustancialmente su participación. Ahora, una mirada a la producción muestra que desde mediados de los años '80 la manufactura ha sido capaz de mantener su parte en el PIB urbano. Esto hace suponer una recuperación de la industria que empezó con la implementación de políticas más flexibles y heterodoxas. Sea como sea, es llamativo que los servicios financieros (incluyendo seguros y servicios de producción) contribuyen más al PIB urbano que la manufactura, siendo el segundo sector tras el comercio. La concentración espacial es incluso más alta que en México: el 78\% del PIB nacional financiero se produce en Santiago, representando el sub-sector más concentrado en Chile (Banco Central de Chile, 2001).

Referente a las ubicaciones de las sedes principales, solamente dos de las 50 empresas más importantes no tienen su sede principal en Santiago, ambas compañías navieras. La distribución regional de IED revela que el área metropolitana de Santiago es lejos la región donde más capital entra (INE, 2002).

Evidencia empírica referente a la tercera condición para la formación de ciudades globales -vinculaciones con otras ciudades globales vía redes de empresas de servicios avanzados de producción y otros flujos críticos- es aportada por estudios de la Globalization and World Cities Study Group and Network (GaWC, Grupo y Red de Estudios de Globalización y Ciudades Globales) que prueban que Ciudad de México está bien incrustada en la red trans-fronteriza de ciudades globales (Taylor, 2004). Entre 55 ciudades globales identificadas por el GaWC, Ciudad de México ocupa el vigésimo lugar, siendo la ciudad latinoamericana con la clasificación más alta. Tiene el 12\% de formación de ciudad global (medido en términos de nivel de oferta de servicios, del sector servicios de producción, en relación con la ciudad con el puntaje más alto), ante São Paulo (con 11\%), Buenos Aires y Caracas (ambos 6\%). Así, Ciudad de México se puede comparar con ciudades como Zurich, Johannesburgo, Milán e incluso Los Angeles. Por su parte, Santiago ocupa el quinto lugar en Latinoamérica con 5\% de formación de ciudad mundial.

\section{Servicios de producción como el enlace faltante}

Referente a este punto, el argumento ha sido que las tres condiciones antes formuladas (la profundización de procesos trans-estatales, la concentración de funciones de mando y control en ambas capitales, y su incrustación en la red de ciudades globales) tienen que cumplirse para probar la formación de una ciudad global. El punto crucial es que, aunque cada uno de los resultados puede estar bien documentado individualmente, los vínculos entre ellos aún no están comprobados. Sin embargo, es exactamente la conexión entre la integración más profunda en la economía mundial, la importancia y concentración creciente de servicios avanzados de producción, y los vínculos trans-fronterizos entre ciudades, la que da una idea del sonido de la formación de ciudades globales. Un camino hacia un mejor entendimiento de cómo se encuentran actividades económicas transnacionales, servicios centralizados y redes trans-fronterizas, es integrar dos ramas de la literatura especializada: la investigación de ciudades globales y la investigación de cadenas globales de producción. A pesar de que ambas analizan redes trans-estatales, existe poca fertilización cruzada entre los dos campos de investigación. Por lo tanto, el papel crucial de empresas de servicios de producción como eslabones de cadenas globales de mercancías y redes urbanas, en gran parte, ha sido descuidado por la investigación de la globalización (Parnreiter, 2003).

El enfoque de cadenas globales de mercancías (para el enfoque teórico y estudios de casos concretos ver, ejemplarmente, Hopkins \& Wallerstein, 1986; Gereffi \& Korzeniewicz, 1994; Gereffi et al., 2001) tiene sus mayores méritos en captar los cambios organizacionales en la producción y el comercio global desde los años '70. Estos son, primero, nuevos modos de outsourcing industrial de componentes anteriormente integrados de actividades de CTN. Segundo, el comercio está creciendo en sub-componentes y servicios, y, consecuentemente, es más complejo que en tiempos anteriores. Tercero, una mayor parte del comercio 
global (más de dos tercios) ahora es conducido dentro de CTN o por sistemas de gobernación que vinculan empresas entre ellas a través de una variedad de acuerdos de selección y contratación (ver, por ejemplo, Dicken, 1998). Trazando todos los input requeridos para llevar un producto desde la concepción, por las fases intermediarias de producción, a los consumidores, el enfoque de la mercancía global realza vinculaciones y coordinaciones entre agentes y lugares económicos. No obstante sus importantes contribuciones conceptuales y un número creciente de estudios de caso empíricos de diferentes mercancías, hay que declarar una limitación seria: a la investigación en este campo le falta el entendimiento del papel crucial de servicios de producción. Este déficit es sorprendente porque las redes trans-fronterizas de producción requieren de formas sofisticadas de logística, coordinación y control. Estos input son provistos por intermediarios de servicios. Empresas de servicios de producción suministran una diversidad de servicios financieros, profesionales y creativos, que son esenciales para la inserción de empresas en todo el mundo en una división de trabajo global. Pese a un llamado inicial de explorar el sector de servicios como vínculo clave dentro de las cadenas globales de mercancías (Rabach \& Kim, 1994), esto campo quedó sub-investigado.

La investigación de ciudades globales, por su parte, tiene su fuerte en la conceptualización de las relaciones entre globalización y ciudades, prestando mucha atención al papel de los servicios avanzados de producción. Ya que servicios intermediarios ya han sido identificados como actores claves en la formación de ciudades globales y en ligar la red de ciudades mundiales, consideramos la función doble de empresas de servicios de producción como proveedores de conectividad tanto para cadenas de mercancías como para redes urbanas.

Con nuestra investigación empírica damos un primer paso en esta dirección, por ejemplo, identificando las empresas de servicios de producción como eslabones de cadenas globales de producción y redes urbanas. Recurriendo a instituciones financieras que proveen servicios a compañías importantes en México y Chile, queremos establecer un mejor entendimiento de cómo una producción espacialmente dispersa, servicios centrales y redes trans-fronterizas se unen. En otras palabras, detectando las empresas de servicios financieros involucrados (sean compañías locales o globales) esperamos comprender cómo y por quién las conexiones entre la producción y las redes urbanas son establecidas y mantenidas.

\section{Globalización y el papel de intermediarios de servicios financieros en México y Chile}

Nos concentramos en el sector financiero porque representa una forma importante de la actividad económica en la cual se puede observar la globalización. En este sentido, la globalización ha conducido a un grado más profundo de integración financiera de países latinoamericanos en el mercado mundial. Por consiguiente, la internacionalización de servicios financieros es un desarrollo importante vinculado a la globalización (Eichengreen, 1996). Esta interiorización es alcanzada vía dos canales (Schmukler, 2004). El primer canal es una mayor presencia de proveedores internacionales del sector financiero, principalmente bancos extranjeros, en los mercados locales. El segundo canal involucra el uso de intermediarios financieros internacionales que se ubican fuera del país, por medio de empresas locales, prestatarios e inversionistas. Una característica de lo último es el comercio de bonos y acciones, por lo general en forma de American Depositary Receipts (ADRs) en bolsas grandes. La internacionalización de las instituciones financieras es estimulada, primero, por ganancias en tecnología de información que ofrece la posibilidad de proveer una gran variedad de productos y servicios financieros en diferentes mercados y países. Segundo, bancos y empresas financieras pretendían enfrentarse a la mayor competencia ampliando sus acciones de mercado hacia negocios y mercados nuevos con el objetivo de diversificar los riesgos. Tercero, la liberalización de los sistemas regulatorios junto con la privatización de instituciones públicas financieras abrió la puerta para que empresas internacionales entren a los mercados locales. Estas tendencias generales otorgaron su camino a los sistemas financieros de México y Chile.

\section{El sector de servicios financieros en México y Chile}

En México, el sistema financiero fue sometido a un importante paquete de reformas después de 
la denominada Crisis del Peso en 1994/95, pero especialmente en 1998/99 cuando los obstáculos restantes a la propiedad extranjera fueron aliviados. Así, el papel del capital extranjero en el proceso de consolidación del sector financiero ha sido significativo: con la liberalización total, la participación extranjera creció a más que tres cuartos del capital fijo bancario en el 2001. Actualmente, los cinco bancos más grandes que representan el $85 \%$ del capital bancario nacional son propiedad de bancos extranjeros (OECD, 2002; Krebsbach, 2003). En Chile, un proceso de consolidación rápido de la industria financiera tenía lugar durante los años '90. El número de bancos bajó de 40 en 1992 a 26 en el 2003, con cuatro bancos alcanzando casi el 70\% de participación en el mercado. En 1997 y 2001 el gobierno levantó varias restricciones para el sistema bancario, abriendo nuevas actividades de negocio para bancos (como inversiones internacionales en bancos, actividades derivadas e investment banking). La liberalización de mercados de capitales incrementó considerablemente la participación de instituciones extranjeras. Actualmente, siete bancos controlados desde el extranjero - encabezados por la española Santander Central Hispano-dominan el sector con una participación en el mercado de $45 \%$, seguidos por ocho bancos bajo control local, el Banco del Estado, nueve sucursales de bancos extranjeros y una compañía de finanzas (Latin America Monitor, 2004; EIU, 2004; SBIF, 2004).

La mayor presencia de bancos extranjeros y la internacionalización resultante de los servicios financieros moldearon el camino para que las compañías mexicanas tengan acceso a capital de inversión. Gigantes financieros globales como BBVA, Citigroup, HSBC y Santander Central Hispano hicieron importantes inversiones (se estima US\$ 25 billones) en el sistema bancario mexicano en los últimos diez años (OECD, 2004, p. 14). Estas inversiones no se hicieron para fomentar créditos particulares, pero pueden ser vistos como una señal para un cambio en las fórmulas de ingresos de los bancos. El mercado al detalle (créditos de consumo e hipotecarios), en gran parte sub-atendido por los bancos, y envíos de dinero de trabajadores generan comisiones altas y por eso prometen beneficios más altos para bancos internacionales que los intereses en préstamos. Por lo tanto, los bancos internacionales que operan en México se concentraron en gran parte en este segmento de mercado más rentable. Mientras intermediarios financieros no-bancarios se hicieron fuente de crédito para empresas pequeñas y medianas, el negocio de créditos corporativos high-end sacó provecho del acceso más fácil a capital internacional debido a la presencia de firmas internacionales de servicios financieros. Por el alto costo de préstamos, la emisión de bonos nacionales e internacionales y la participación de compañías en el mercado de valores de EE.UU. usando ADRs han crecido de manera significativa. La tasa media de comercio de ADR en Nueva York sobre el valor total comercializado en los mercados mexicanos aumentó desde alrededor de 30\% (1990-1995) a 70\% (1996-1999) (Schmuckler, 2004, Cuadro 4, p. 47).

En Chile, los créditos de consumo y los préstamos hipotecarios de los bancos registran tasas de crecimiento considerables, mientras el financiamiento corporativo creció solo $1 \%$ (todos los datos 2003, EIU, 2004). Los bancos grandes y extranjeros en Chile dan créditos casi exclusivamente a empresas grandes y multinacionales, y se concentran en actividades del mercado de capitales. En concordancia, midiendo los diferentes sectores del sistema financiero, Gallego y Loayza llegan a la conclusión que el proceso de liberalización se ha relacionado con un cambio en la estructura de la economía, de tal manera que los mercados de acciones y otros capitales ganaron importancia en relación con el sector bancario (Gallego \& Loayza, 1999, pp. 18-21). De esta manera, a fines de la década de los '90, los mercados locales de acciones y bonos han sobrepasado el mercado de créditos en actividad, tamaño y rendimiento (OECD, 2003).

\section{Base empírica y método}

Para comprender la importancia de instituciones financieras que operan en Chile y México dependemos de dos fuentes de datos. El primer conjunto de datos, compilado por el grupo GaWC en 1997/98, consiste en una matriz de valores de servicio de 100 empresas globales de servicios en los sectores de contabilidad, publicidad, derecho, seguros, consultoría en gestión y servicios financieros. Los valores de servicio son estimaciones 
de la importancia de una ciudad dentro de la estrategia global de oficinas de una empresa, tomando en cuenta características como el tamaño y la función de las oficinas. A causa del carácter multifacético de los datos, extraídos en gran parte de las páginas Web de las empresas, los valores de servicio se basan en un código simple con valores entre 0 (sin presencia) y 5 (sedes principales). Este análisis define las relaciones entre ciudades por medio de la presencia diferenciada de empresas de servicios globales en cada ciudad, usando la presunción plausible que cuanto más grande el valor de servicio de una empresa (el nivel de servicios ofrecidos por esta empresa) en una ciudad, más grande la cantidad de flujos de información, conocimientos, instrucción, ideas, estrategias, planes, etc. que irradiará una empresa desde esta ciudad para conectarse con oficinas en otras ciudades (para detalles ver Taylor, 2004). Recurrimos a los resultados referentes a los proveedores de servicios financieros.

La segunda fuente de información es la base de datos de Bloomberg. Esta red de informaciones financieras fue lanzada en 1982 y provee información sobre cómo las compañías se auto-financian declarando la emisión de acciones y obligaciones por compañía, la(s) bolsa(s) donde estos instrumentos están cotizados, y, en la mayoría de los casos, el (los) nombre(s) de la(s) empresa(s) involucrada(s) en la transacción. Esto nos permite derivar una relación directa entre compañías y la empresa de servicios financieros involucrada (para información en detalle ver http://www. bloomberg.com).

Recurriendo a la base de datos de Bloomberg, analizamos la emisión de bonos y acciones de las 50 compañías financieras y no-financieras más importantes en México y Chile clasificadas por ventas (Expansión, 2002; El Diario, 2003). Entre estas compañías, en ambos países se encuentran tanto empresas que producen principalmente para el mercado global (p.e. Petróleos Mexicanos, Codelco) como empresas orientadas preferentemente hacia el mercado interno (p.e. Teléfonos de México, Telefónica CTC Chile). Después de identificar los proveedores de servicios financieros que atienden a los top 50, los clasificamos por frecuencia de ocurrencia. Como segunda etapa, comparamos nuestros hallazgos en Bloomberg con los resultados de GaWC. Además de enseñar información referente a la presencia y significancia de los proveedores de servicios financieros en México y Chile, estamos en condiciones de establecer cambios comparando nuestros resultados con los del grupo GaWC.

\section{Resultados}

Referente a la muestra mexicana, en el caso de 26 empresas de las 50 más importantes estamos en condiciones de identificar en la base de datos de Bloomberg las empresas de servicios financieros que fueron involucradas, ya sea en una emisión de acciones o de obligaciones. En el caso de doce compañías que figuraron como parte de los top 50, la filial mexicana no apareció en el mercado nacional de acciones/bonos. Esto lleva a la suposición que se financian internamente (por ejemplo emisiones de acciones/obligaciones de las sedes principales ubicadas fuera de México). En algunos casos, la compañía matriz extranjera apareció cotizada en la bolsa mexicana pero no la sucursal mexicana. Se restaron estas compañías de la muestra, porque buscamos la relación entre un proveedor de servicio y empresas operando en México. Adicionalmente, se excluyeron tres instituciones financieras que han sido des-cotizadas del mercado mexicano de acciones después de la toma de posesión extranjera, más el Banco Estatal.

En cuanto a Chile, en 20 de las 50 compañías más importantes estamos en condiciones de identificar las instituciones financieras que atienden sus transacciones de bolsa. En los demás casos, la base de datos de Bloomberg no revela información de los intermediarios de servicio involucrados.

\section{Clasificación de Asset Managers en México y en Chile}

El ranking de compañías de servicios financieros por la frecuencia de su ocurrencia en la base de datos de Bloomberg, se muestra en el cuadro 1 para México y en cuadro 2 para Chile (la clasificación GaWC se reproduce como comparación).

En el caso de México, la Citibank/Citigroup (a la cual agregamos Salomon Smith Barney y Banamex por sus adquisiciones en 1998 y 2001, 
Cuadro 1. México - Clasificación de proveedores de servicios financieros

\begin{tabular}{|c|c|c|c|c|}
\hline $\begin{array}{l}\text { Institución } \\
\text { Financiera }\end{array}$ & $\begin{array}{c}\text { Ranking } \\
\text { Bloomberg } \\
\text { (transacciones } \\
\text { por frecuencia } \\
\text { de ocurrencia) }\end{array}$ & $\begin{array}{l}\text { Clasificación } \\
\text { GaWC } \\
(0-5)\end{array}$ & $\begin{array}{l}\text { Cliente } \\
\text { (Clasificación nacional de la } \\
\text { compañía por ventas) }\end{array}$ & $\begin{array}{l}\text { Oficina en } \\
\text { México } \\
(2004)\end{array}$ \\
\hline $\begin{array}{l}\text { Citibank / Citigroup } \\
\text { (con Salomon Smith } \\
\text { Barney y Banamex } \\
\text { agregado) }\end{array}$ & 15 & $\begin{array}{l}2 \text { Citibank } \\
- \text { Salomon }\end{array}$ & $\begin{array}{l}\text { Petróleos Mexicanos (1), Teléfonos de } \\
\text { México (3), Grupo Carso (6), Walmart } \\
\text { de México (8), Cemex (12), Controlada } \\
\text { Comercial Mexicana (19), Savia (21) } \\
\text { Vitro (25), Grupo Televisa (34), Gruma } \\
\text { (38), Kimberly Clark de México (42), El } \\
\text { Puerto de Liverpool (43) Grupo Bimbo (20), } \\
\text { Grupo IMSA (33), Coca-Cola Femsa (40) }\end{array}$ & sí \\
\hline Bank of New York & 12 & - & $\begin{array}{l}\text { Carso Global Telecom (2), Grupo Carso } \\
\text { (6), Walmart de México (8), Fomento } \\
\text { Económico Mexicano (13), Controlada } \\
\text { Comercial Mexicana (19), Vitro (25) } \\
\text { Grupo Gigante (26), Grupo Televisa (34), } \\
\text { FEMSA Cerveza (37), Coca Cola Femsa (40), } \\
\text { Grupo Casa Saba (44), Hylsamex (49) } \\
\end{array}$ & sí \\
\hline $\begin{array}{l}\text { JP Morgan Chase } \\
\text { (fusionado en 2000) }\end{array}$ & 12 & $\begin{array}{l}3 \text { JPM } \\
2 \text { Chase }\end{array}$ & $\begin{array}{l}\text { Petróleos Mexicanos (1), Teléfonos de México } \\
\text { (3), Walmart de México (8), Cemex (12), Grupo } \\
\text { Bimbo (20), Savia (21), Grupo Financiero } \\
\text { Banamex Accival (Nassau Branch) (29), Grupo } \\
\text { Televisa (34), Gruma (38), Coca-Cola Femsa } \\
\text { (40), Grupo Casa Saba (44), Nadro (45) }\end{array}$ & sí \\
\hline ING Bank & 9 & - & $\begin{array}{l}\text { Petróleos Mexicanos (1), } \\
\text { Teléfonos de México (3), Volkswagen de } \\
\text { México (9), Cemex (12), Controlada Comercial } \\
\text { Mexicana (19), Grupo Bimbo (20), Grupo } \\
\text { IMSA (33), Grupo Televisa (34), Savia (21) }\end{array}$ & sí \\
\hline Deutsche Bank & 7 & 0 & $\begin{array}{l}\text { Petróleos Mexicanos (1), Teléfonos de México } \\
\text { (3), Walmart de México (8), Cemex (12), } \\
\text { Savia (21), Grupo Financiero Banamex } \\
\text { Accival (29), Grupo Televisa (34) }\end{array}$ & sí \\
\hline $\begin{array}{l}\text { Credit Suisse } \\
\text { First Boston }\end{array}$ & 5 & 2 & $\begin{array}{l}\text { Petróleos Mexicanos (1), Teléfonos de } \\
\text { México (3), Vitro (25), Grupo Financiero } \\
\text { Banamex Accival (29), Grupo Televisa (34) }\end{array}$ & sí \\
\hline Bear Stearns & 4 & - & $\begin{array}{l}\text { Petróleos Mexicanos (1), Gruma } \\
\text { (38), Coca-Cola Femsa (40) }\end{array}$ & no \\
\hline Inversora Bursátil & 4 & & $\begin{array}{l}\text { Teléfonos de México (3), Grupo Carso (6), } \\
\text { Grupo Alfa (14), Grupo Televisa (34) }\end{array}$ & sí \\
\hline Lehman Brothers & 3 & - & $\begin{array}{l}\text { Petróleos Mexicanos (1), Gruma } \\
\text { (38), Aerovías de México (47) }\end{array}$ & sí \\
\hline Merryll Lynch & 3 & - & $\begin{array}{l}\text { Teléfonos de México (3), Grupo Financiero } \\
\text { Banamex Accival (29), Grupo Televisa (34) } \\
\end{array}$ & sí \\
\hline Santander Serfin & 3 & - & Vitro (25), Cemex (12), Hylsamex (49) & sí \\
\hline $\begin{array}{l}\text { Dexia Banque } \\
\text { International á } \\
\text { Luxembourg }\end{array}$ & 2 & - & $\begin{array}{l}\text { Grupo Televisa (34), Kimberly } \\
\text { Clark de México (42) }\end{array}$ & sí \\
\hline $\begin{array}{l}\text { Kredietbank } \\
\text { Luxembourgeoise }\end{array}$ & 2 & - & Teléfonos de México (3), Savia (21) & no \\
\hline $\begin{array}{l}\text { Morgan Stanley } \\
\text { Dean Witter }\end{array}$ & 2 & - & $\begin{array}{l}\text { Grupo Alfa (14), Grupo Financiero } \\
\text { Banamex Accival (29) }\end{array}$ & sí \\
\hline Scotia Inverlat & 2 & - & Vitro (25), Kimberly Clark de México (42) & sí \\
\hline UBS & 2 & 0 & Petróleos Mexicanos (1), FEMSA Cerveza (37) & sí \\
\hline
\end{tabular}




\begin{tabular}{|c|c|c|c|c|}
\hline ABN AMRO & 1 & 2 & Petróleos Mexicanos (1) & sí \\
\hline $\begin{array}{l}\text { Afin Casa de Bolsa } \\
\text { (Grupo Financiero } \\
\text { Banorte) }\end{array}$ & 1 & & Gruma (38) & sí \\
\hline $\begin{array}{l}\text { Banca Nazionale } \\
\text { de Lavoro }\end{array}$ & 1 & - & Petróleos Mexicanos (1) & no \\
\hline $\begin{array}{l}\text { Banco Commerciale } \\
\text { Italia (banca intesa) }\end{array}$ & 1 & - & Petróleos Mexicanos (1) & no \\
\hline $\begin{array}{l}\text { Bank of America } \\
\text { México }\end{array}$ & 1 & - & Grupo Bimbo (20) & sí \\
\hline Barclays Capital & 1 & 3 & Petróleos Mexicanos (1) & no \\
\hline $\begin{array}{l}\text { BMO Nesbit Burns } \\
\text { International }\end{array}$ & 1 & - & Savia (21) & sí \\
\hline $\begin{array}{l}\text { Caboto SIM Spa } \\
\text { (Banca Intesa) }\end{array}$ & 1 & - & Petróleos Mexicanos (1) & no \\
\hline Cassa di Risparmio & 1 & - & Petróleos Mexicanos (1) & no \\
\hline $\begin{array}{l}\text { Dresdner Kleinwort } \\
\text { Wasserstein SEC }\end{array}$ & 1 & - & Grupo Televisa (34) & no \\
\hline Goldman Sachs & 1 & - & Petróleos Mexicanos (1) & sí \\
\hline HSBC & 1 & 2 & Petróleos Mexicanos (1) & sí \\
\hline $\begin{array}{l}\text { IBJ Schroder } \\
\text { Bank \& Trust } \\
\text { (ahora IBJ Whitehall } \\
\text { Trust Company) } \\
\end{array}$ & 1 & - & Controlada Comercial Mexicana (19) & no \\
\hline $\begin{array}{l}\text { Lazard Freres } \\
\text { Luxembourg }\end{array}$ & 1 & - & El Puerto de Liverpool (43) & no \\
\hline $\begin{array}{l}\text { Multivalores } \\
\text { Casa de Bolsa }\end{array}$ & 1 & & BBVA Bancomer (36) & sí \\
\hline $\begin{array}{l}\text { Standard Bank } \\
\text { London }\end{array}$ & 1 & - & Vitro (25) & sí \\
\hline US Bank Trust & 1 & - & Cemex (12) & no \\
\hline Value Casa de Bolsa & 1 & & Vitro (25) & sí \\
\hline
\end{tabular}

Fuente. Elaboración propia

respectivamente) encabezan la firmas de servicios financieros, seguidos por JP Morgan Chase y Bank of New York, esta última principalmente por su papel protagonista como depósito de los ADR. Revisando las firmas de servicios financieros detectados, una característica principal llama la atención: la mayoría es propiedad extranjera: solo cuatro de 34 proveedores de servicios financieros son propiedad de capital mexicano (en letra cursiva en los cuadros 1 y 2 ).

Por lo que a la presencia de instituciones financieras extranjeras se refiere, la imagen en Chile es similar a la de México. Solamente cuatro de 26 instituciones financieras detectadas son propiedad de capital privado chileno (IM Trust se convirtió en $100 \%$ chileno después de abandonar la socie- dad con Bankers Trust en 1992). Proveedores de servicios con alcance global encabezan la clasificación: Citigroup comparte la primera posición con JP Morgan Chase Manhattan, seguidos por el español Banco Santander y por Morgan Stanley Dean Witter. A diferencia de México, algunas instituciones extranjeras con una alta frecuencia de ocurrencia no mantienen una oficina local, incluso cuando son más importantes que sus contrapartes en México (esto se refiere a Morgan Stanley, Bank of New York y Dresdner Kleinwort Wasserstein).

\section{Evaluación empirica: comparando los datos de Bloomberg y GaWC}

Una comparación de los datos de Bloomberg y de GaWC revela cambios sustanciales referentes a 
Cuadro 2. Chile - Clasificación de proveedores de servicios financieros

\begin{tabular}{|c|c|c|c|c|}
\hline $\begin{array}{l}\text { Institución } \\
\text { Financiera }\end{array}$ & $\begin{array}{c}\text { Ranking } \\
\text { Bloomberg } \\
\text { (transacciones } \\
\text { por frecuencia } \\
\text { de ocurrencia) }\end{array}$ & $\begin{array}{l}\text { Clasificación } \\
\text { GaWC } \\
(0-5)\end{array}$ & $\begin{array}{l}\text { Cliente } \\
\text { (Clasificación nacional de la } \\
\text { compañía por ventas) }\end{array}$ & $\begin{array}{l}\text { Oficina en } \\
\text { Chile (2004) }\end{array}$ \\
\hline $\begin{array}{l}\text { Citibank / Citicorp } \\
\text { Chile (con Salomon } \\
\text { Smith Barney } \\
\text { agregado) }\end{array}$ & 6 & $\begin{array}{l}- \text { Salomon } \\
3 \text { Citibank }\end{array}$ & $\begin{array}{l}\text { Codelco (1), Endesa (10), Telefónica CTC } \\
\text { Chile (12), Minera Escondida (18), SQM } \\
\text { (24), Citibank (49) }\end{array}$ & sí \\
\hline $\begin{array}{l}\text { JP Morgan Chase } \\
\text { Manhattan } \\
\text { (fusionado en 2000) }\end{array}$ & 6 & $\begin{array}{l}2 \text { JPM } \\
2 \text { Chase }\end{array}$ & $\begin{array}{l}\text { Codelco (1), ENAP (5), Banco Santander (6), } \\
\text { Endesa (10), CTC (12), CMPC (16) }\end{array}$ & sí \\
\hline $\begin{array}{l}\text { Banco Santander } \\
\text { / Santander } \\
\text { Investment Bank } \\
\text { / Santander } \\
\text { Corredores }\end{array}$ & 5 & - & $\begin{array}{l}\text { Banco Santander (6), Endesa (10), Banco de } \\
\text { Chile (14), Entel (15), CorpBanca (48) }\end{array}$ & sí \\
\hline $\begin{array}{l}\text { Morgan Stanley } \\
\text { Dean Witter }\end{array}$ & 5 & - & $\begin{array}{l}\text { Codelco (1), Enersis (2), Banco Santander (6), } \\
\text { Endesa (10), CMPC (16) }\end{array}$ & no \\
\hline $\begin{array}{l}\text { Banco Bice / Bice } \\
\text { Corredores de Bolsa }\end{array}$ & 4 & & $\begin{array}{l}\text { CTC (12), Sodimac (21), CCU (30), Gasco } \\
(43)\end{array}$ & sí \\
\hline $\begin{array}{l}\text { Deutsche Bank } \\
\text { Securities }\end{array}$ & 4 & 3 & $\begin{array}{l}\text { Enersis (2), ENAP (5), Endesa (10), AES } \\
\text { Gener (28) }\end{array}$ & sí \\
\hline $\begin{array}{l}\text { BBVA (Banco Bilbao } \\
\text { Vizcaya Argentaria) }\end{array}$ & 4 & - & $\begin{array}{l}\text { Codelco (1), Enersis (2), Endesa (10), } \\
\text { Cencosud (17) }\end{array}$ & sí \\
\hline Bank of New York & 3 & - & Cencosud (17), BBVA (41), CorpBanca (48) & no \\
\hline $\begin{array}{l}\text { IM Trust \& Co / IM } \\
\text { Trust Corredores }\end{array}$ & 3 & & CTC (12), Sodimac (21), CorpBanca (48) & sí \\
\hline $\begin{array}{l}\text { Dresdner Kleinwort } \\
\text { Wasserstein } \\
\text { Securities }\end{array}$ & 2 & - & Codelco (1), Endesa (10) & no \\
\hline Merrill Lynch & 2 & - & Codelco (1), BBVA (41) & sí \\
\hline BNP Paribas & 2 & 2 & Codelco (1), Endesa (10) & sí \\
\hline $\begin{array}{l}\text { American } \\
\text { Express Bank }\end{array}$ & 1 & - & CTC (12) & sí \\
\hline Banca IMI & 1 & - & Endesa $(10)$ & no \\
\hline $\begin{array}{l}\text { Banca Nazionale } \\
\text { del Lavoro }\end{array}$ & 1 & - & Endesa (10) & no \\
\hline Banco de Chile & 1 & & Banco de Chile (14) & sí \\
\hline $\begin{array}{l}\text { Bank of America } \\
\text { Securities }\end{array}$ & 1 & - & Endesa (10) & sí \\
\hline BCI & 1 & & BCI (22) & sí \\
\hline Credit Lyonnais & 1 & - & Endesa (10) & sí \\
\hline $\begin{array}{l}\text { Credit Suisse } \\
\text { First Boston }\end{array}$ & 1 & 0 & CorpBanca (48) & no \\
\hline DF King & 1 & - & Banco Santander (6) & no \\
\hline $\begin{array}{l}\text { Goldman } \\
\text { Sachs \& Co }\end{array}$ & 1 & - & BBVA (41) & no \\
\hline HSBC & 1 & 2 & Endesa (10) & sí \\
\hline ING Barings & 1 & 3 & Endesa (10) & sí \\
\hline $\begin{array}{l}\text { RBC Capital } \\
\text { Markets }\end{array}$ & 1 & - & Codelco (1) & no \\
\hline UBS & 1 & 0 & Endesa (10) & sí \\
\hline
\end{tabular}

Fuente. Elaboración propia 
la presencia de compañías bancarias y financieras en México y Chile, y en la estructura del sector bancario mismo. En el caso de México, lo más notable es que los bancos japoneses perdieron fondo. Mientras muestran un valor de servicio alto en la matriz de GaWC, nuestro conjunto de datos reveló que no estaban en absoluto involucrados en la emisión de acciones o bonos de compañías mexicanas. Incluso, referente a su presencia en el mercado mexicano, solamente tres de cinco bancos que estaban incluidos en la lista de GaWC siguen con una filial en México. Con respecto a los bancos alemanes, nuestros resultados también son opuestos a los de la matriz de GaWC. Deutsche Bank/Bankers Trust (valor de servicio 0 en la lista GaWC) está muy presente y llega a ser quinto en la emisión de acciones/bonos. Todos los demás bancos alemanes de la lista GaWC se volvieron menos importantes: la Bayerische Landesbank Girozentrale incluso cerró su filial local. Instituciones financieras estadounidenses y españolas, sin embargo, extendieron su participación.

En el caso de Chile, uno de los cuatro bancos con el valor de servicio más alto en el estudio de GaWC recientemente desapareció como una entidad independiente: Dresdner Bank reestructuró su negocio en latinoamerica y vendió sus filiales al Chilean Group Security en abril del 2004. Usando el valor de servicio de instituciones de servicios de GaWC y su frecuencia de ocurrencia en la base de datos de Bloomberg vía comparación, la Deutsche Bank muestra una presencia actual; las instituciones financieras estadounidenses Citibank y JP Morgan Chase incluso podían ampliar su participación. La otra mitad de proveedores de servicios clasificados en la lista GaWC muestran un índice mas bajo en nuestra investigación. Sorprende que cuatro firmas clasificadas en GaWC (WestLB, Rabobank International, Barcleys y BTM) no aparezcan como asset managers: British Barclay en este momento ni mantiene una oficina en Santiago. British HSBC y las holandesas Rabobank, ABM AMRO e ING redujeron sus actividades vendiendo partes de sus filiales locales a otros bancos (Estrategia, 2002). El Bank of New York trabaja exclusivamente como depósito de ADRs y no mantiene una presencia directa en Chile (por esto el banco no fue registrado en el ranking GaWC, igual a Morgan Stanley Dean
Witter). Como en el caso de México, el predominio regional de bancos españoles no fue revelado por la investigación de GaWC.

\section{Interpretación de los hallazgos empíricos}

La investigación muestra dos resultados principales. Primero, que son sobre todo instituciones financieras que operan a nivel global las que se dedican a transacciones de bolsa por encargo de grandes empresas que operan en México y Chile. Cabe destacar que la preferencia por instituciones financieras globales aumenta con la "globalidad" de la empresa chilena o mexicana (p.e. en cuanto al volumen de las exportaciones y las importaciones, o al valor de bonos o acciones emitidos en mercados extranjeros).

Segundo, y más importante, la investigación revela que entre las instituciones financieras globales las más preferidas por las grandes compañias operando en México y en Chile son las que mantienen oficinas locales en Ciudad de México y Santiago. Esta tendencia se muestra tanto para empresas que producen hacia el mercado global como para compañías con una orientación más nacional. En ambos casos, la demanda por servicios financieros se enfoca en instituciones financieras operando en escalas distintas. Por lo tanto, el lema central es "glocalización", lo que se refleja en la publicidad de grandes bancos. Según UBS, el banco actúa "in two locations: everywhere, and right next to you", mientras HSBC se califica como "The world's local bank". Se puede concluir, entonces, que ambas ciudades (Ciudad de México y Santiago) son lugares importantes en las estrategias geográficas de bancos como Citibank / Citigroup, JP Morgan Chase, Bank of New York, ING Bank o Banco Santander. Eso coincide con los resultados de GaWC y con investigaciones recientes sobre la formación de ciudades globales en América Latina.

Entre los clientes de las instituciones financieras "glocales" se encuentran las empresas más grandes de México y de Chile, que, en muchos casos, producen para el mercado global. Cuando el mercado doméstico representa el enfoque principal (como, p.e., en el caso de las compañías de telecomunicación), la relación con el mercado 
global se establece a través de inversiones extranjeras. La investigación muestra que desde la Ciudad de México y desde Santiago se prestan servicios importantes para el funcionamiento de las cadenas de producción, que relacionan la producción en México y en Chile con el mercado global. Y, al revés: las empresas grandes operando en México y en Chile que demandan servicios de instituciones financieras "glocales" contribuyen a la transformación de la Ciudad de México y Santiago en ciudades globales.

La preferencia por instituciones financieras "glocales" se puede atribuir a dos factores. Primero, las compañías grandes en México y en Chile buscan instituciones financieras con suficiente experiencia y conocimiento en la emisión de bonos $\mathrm{y}$ acciones en mercados financieros internacionales (principalmente los mercados de Nueva York, Luxemburgo y Francfort). Sin embargo, ya que varios bancos ofrecen una experiencia similar, las compañías se dirigen al banco que esté más cerca, es decir, en Ciudad de México o en Santiago. Por eso, para las instituciones financieras globales es, como advierte el director ejecutivo de la oficina mexicana de uno de estos bancos, "importantísimo tener presencia geográfica para captar el mercado". El segundo motivo para el predominio de instituciones financieras que operan tanto a nivel global como a nivel local, se deduce del hecho que entre las compañías analizadas se encuentran muchas empresas afiliadas de CTNs. Para la sucursal mexicana de una CTN, por ejemplo, es evidente escoger un banco que ya está encargado de las transacciones financieras de la sede en Nueva York.

Aunque el análisis realizado no permite una conclusión definitiva sobre el tipo de servicios prestados desde Ciudad de México y Santiago, se pueden indicar los primeros rasgos de la división de trabajo entre la oficina local y la casa matriz en Nueva York u otras ciudades globales. Una transacción se puede separar en tres etapas: primero, el "sourcing", es decir, la producción del "deal"; segundo, la preparación de la transacción y su ejecución; y, tercero, la venta de las acciones o bonos. Las oficinas en Ciudad de México o en Santiago son responsables de los dos primeros pasos, en los que "debes estar cerca del cliente", como dice el director ejecutivo ya citado. Sólo la presencia local permite a las instituciones financieras saber (o, quizá, predecir) qué empresa está pensando en emitir acciones en el futuro próximo, clave para poder generar los "deals". Además, solo a través de la presencia local se logra un conocimiento detallado tanto de la empresa como de la economía nacional. Ambos pasos son, sin embargo, cruciales para evaluar si una transacción puede tener éxito. En la producción y en la preparación de una transacción la oficina local asume toda la responsibilidad, tal vez apoyada por especialistas viniendo de Nueva York o Londres (p.e. para industrias particulares). Sin embargo, la oficina en Ciudad de México o en Santiago necesita la autorización de las transacciones por parte de la casa matriz del banco.

Esta supuesta división de trabajo entre los proveedores de servicios financieros concuerda con el análisis de Taylor (2004): aunque la red de ciudades globales incluya ciudades en países periféricos o semi-periféricos, la red no se constituye por nodos homogénos. Existen diferencias importantes entre ciudades en cuanto a su capacidad de mando y control, diferencias que se ha discutido con más detalle, en cuanto a la Ciudad de México, en Parnreiter (2002). Así, el alcance de la Ciudad de México como ciudad global no traspasa México, ya que Miami sigue siendo el centro regional indiscutiblemente. Además, en cuanto a la integración de México en la economía global, Miami no está involucrada. En este caso, es en Ciudad de México donde se organiza y gestiona la articulación de una economía regional con la economía global. Existen, entonces, diferencias importantes entre las ciudades, lo que nos obliga a conceptualizar la desigualdad. Luego, los términos "centro" y "periferia" no han perdido su valor explicativo, a pesar de las transformaciones profundas por las cuales pasó el sistema mundial. En la era de la globalización, la producción y reproducción de "centro" y "periferia" se da en una escala cada vez más grande y pequeña. Eso hace complejo indicar el lugar exacto de la Ciudad de México o de Santiago en la jerarquía urbana global. Por un lado, es evidente que, por ejemplo, la Ciudad de México no es un centro global. Pero, por el otro, sí comprende áreas, personas y actividades que pertenecen a los "flujos de la centralidad", es decir, al centro global que se extiende en la red de 
las ciudades globales. Tal vez se puede llamar a la Ciudad de México una "ciudad global relé", orientada hacia y dominada por una o dos ciudades globales, y relacionada con otras "ciudades globales relé". Es en este sentido que Rossi, Beaverstock y Taylor (2007) distinguen entre "decision cities" y "service cities".

Para el propósito de este artículo se puede resumir que son los intermediarios de servicios financieros los que establecen la relación entre cadenas globales de producción y la red de ciudades globales. Citibank/Citigroup, por ejemplo, es una de las instituciones financieras "glocales" que funciona como creador de redes, proporcionando conectividad tanto para cadenas de producción como para redes urbanas. Por un lado, Citibank/ Citigroup relaciona la Ciudad de México y Santiago de Chile con otras ciudades globales a través de los flujos de servicios. Al mismo tiempo, Citibank/ Citigroup presta servicios financieros a empresas de los sectores primarios, secundarios y terciarios de la economía mexicana y chilena, agregando, de esta manera, valor a varias cadenas de producción. Dado que prácticamente toda producción require servicios financieros, se establece, en términos más generales, que cadenas de producción siempre pasan por ciudades globales, mientras todas las ciudades globales están integradas por múltiples cadenas de producción.

Nuestro estudio de las actividades de proveedores de servicios financieros, para los casos de Ciudad de México y de Santiago de Chile, entrega los primeros detalles de cómo y por quién son establecidas y mantenidas las conexiones entre la producción y las redes urbanas. Investigaciones posteriores en esta área ayudarán a especificar cómo las dos redes trans-estatales basadas en empresas se relacionan, y, por lo tanto, proveerán un entendimiento más exhaustivo de la relación entre cambios urbanos y procesos de globalización en Latinoamérica. A nuestro parecer, es el tratamiento integral de empresas de servicios de producción (como creadores de redes), de cadenas de producción (como modalidad organizacional dominante de la producción globalmente dispersa) y de ciudades (como lugares donde la red ocurre) lo que nos permite comprender las transformaciones estructurales de los últimos treinta años.

\section{Referencias bibliográficas}

Banco Central de Chile (2001). Indicadores económicos y sociales de Chile 1960-2000. Santiago: Banco Central.

Dicken, P. (1998). Global shift: transforming the world economy ( $3^{\text {ra }}$ ed.). London: Paul Chapman Publishing.

Dussel Peters, E. (2000). Polarizing Mexico. The impact of liberalization strategy ( $1^{\mathrm{a}} \mathrm{ed}$.). Boulder/London: Lynne Rienner Publishers.

Eichengreen, B. (1996). Globalizing capital (1 ${ }^{\text {a }}$ ed.). Princeton: Princeton University Press.

EIU/ Economist Intelligence Unit (2004). Chile country profile. Recuperado el 24 de enero de 2005, de www.eiu.com.

El Diario (2003, 28 de marzo). Resultados de empresas.

Estrategia (2002). Ausencia de grandes bancos de Japón y Europa: el Talón de Aquiles del Sistema. 12.8.2002.

Expansión (2002). Las empresas más importantes de México (CD-Rom). México D.F.

Fazio, H. (2000). La transnacionalización de la economía chilena. Mapa de la extrema riqueza al año 2000 (1 $1^{\mathrm{a}}$ ed.). Santiago de Chile: LOM.

Gallego, F. \& Loayza, N. (1999). Financial structure in Chile: macroeconomic developments and microeconomic effects. Working Papers Central Bank of Chile, 75.

Gereffi, G. \& Korzeniewicz, M. (Eds.) (1994). Commodity chains and global capitalism. Westport: Praeger.

Gereffi, G., Humphrey, J., Kaplinsky, R. \& Sturgeon, T. (Eds.) (2001). The value of value chains: spreading the gains from globalization. IDS Bulletin, 32, 3.

Gwynne, R. (1999). Globalization, commodity chains and fruit exporting regions in Chile. Tijdschrift voor Economische en Sociale Geografie, 90, 2, 211-225.

Hopkins, T. \& Wallerstein, I. (1986). Commodity chains in the world-economy prior to 1800 . Review, 10, 1, 157-170.

Instituto Nacional de Estadísticas, INE (2002). Censo 2002. Recuperado el 24 de enero de 2005, de www.censo2002.cl/.

Krebsbach, K. (2003). The new Mexican revolution. US Banker, 113, 11, 67-69. 
Latin America Monitor (2004). Banking \& Insurance. Southern Cone Monitor, 21,6, 2-7. OECD (2002). Challenges in the Mexican financial sector. Economics Department Working Papers, 339. OECD.

(2003). Chile survey 2003. París:

(2004). México economic survey 2004. Paris: OECD.

Parnreiter, C. (2002). México: the making of a global city? En Saskia Sassen (Ed.), Global networks, linked cities (1 ${ }^{\mathrm{a}}$ ed., pp. 145-182). London: Routledge.

(2003). Global city formation in Latin America: socioeconomic and spatial transformations in Mexico City and Santiago de Chile. GaWC Research Bulletin, 103.

Rabach, E. \& Kim, E.M. (1994). Where is the chain in the commodity chains? The service sector nexus. En G. Gereffi \& Korzeniewicz, M. (Eds.), Commodity chains and global capitalism (1 $1^{\text {a }}$ ed., pp. 123-142). Westport: Praeger.
Rossi, E. C., Beaverstock, J. V. \& Taylor, P. J. (2007) Transaction links through cities: 'decision cities' and 'service cities' in outsourcing by leading Brazilian firms. Geoforum 38, 4, 628-642.

Sassen, S. (1991). The global city. New York, London, Tokyo. Princeton: Princeton University Press.

Superintendencia de bancos e instituciones financieras de Chile, SBIF (2004). Información Financiera. Recuperado el 24 de enero de 2005, de http://www.sbif.cl/

Schmukler, S. (2004). Financial globalization: gain and pain for developing countries. Economic Review, 89, 2, 39-66.

Taylor, P. (2004). World city network: a global urban analysis. London: Routledge.

The Economist Intelligence Unit (2004). Country profile Chile 2004. Recuperado el 24 de enero de 2005, de www.eiu.com. 